\title{
Kinetics and mechanism for the reaction of 1-chloro-2,4- dinitrobenzene with $n$-butylamine and piperidine in AOT/n-hexane/water reverse micelles
}

\author{
Lilian Zingaretti, Liliana Boscatto, Stella. M. Chiacchiera, and Juana J. Silber* \\ Departamento de Química, Universidad Nacional de Río Cuarto, Agencia Postal Nro 3, \\ 5800 Río Cuarto, Argentina \\ E-mail : jsilber@exa.unrc.edu.ar
}

Dedicated to Professor Dr. Edmundo Rúveda and Professor Dr. Roberto A. Rossi

(received 08 Jul 03; accepted 02 Sep 03; published on the web 05 Sep 03)

\begin{abstract}
The kinetic profile of the $S_{N} A r$ reaction of 1-chloro-2,4-dinitrobenzene with n-butylamine and piperidine in $\mathrm{AOT} / \mathrm{n}$-hexane/water reverse micelles were investigated as a function of variables such as AOT and amine concentration and the amount of water dispersed in the reverse micelles, $\mathrm{W}_{\mathrm{o}}=\left[\mathrm{H}_{2} \mathrm{O}\right] /[\mathrm{AOT}]$. The reactions in the micellar medium are faster than in the pure solvent. The pseudo-first order rate constants of the reactions go through a maximum when the AOT concentration is increased. Micellar interface saturation and no further micellar catalysis occurs at c.a. $0.1 \mathrm{M}$ of AOT. At higher AOT concentration dilution of the reactants in the micelles is the responsible of the rate decrease. In micellar medium a significant lost in the catalysis is observed compare with pure $n$-hexane. Thus, the reactions effectively take place at the interface of the aggregates. The kinetic behavior can be quantitatively explained taking into account the distribution of the substrate and the nucleophile between the bulk solvent and the micelle interface. A plausible mechanism to explain the results is proposed. Both the reactants distribution constants, between the micellar pseudo-phase and organic solvent, and the intrinsic second-order rate coefficients of $\mathrm{S}_{\mathrm{N}} \mathrm{Ar}$ reactions in the interface were evaluated.
\end{abstract}

Keywords: Reverse micelles, nucleophilic aromatic substitution, AOT, kinetics

\section{Introduction}

The reactivity of bimolecular reactions is affected by reverse micelles mainly through the increase in local reactant concentrations within the small micellar volume, but also through changes in the rate limiting transition state energy. Therefore, the magnitude of the catalysis depends upon the incorporation of both reactants into the micelle and the reaction rate constant 
in the micellar pseudophase. Hydrophobic interactions between the polar micellar pseudophase with the polar molecule dipoles are the main reason responsible for the reactant micellar incorporation. ${ }^{1,2}$

Among the surfactants that form reverse micelles, the best-known are systems derived from the anionic surfactant Aerosol-OT, (sodium bis (2-ethylhexyl) sulfosuccinate, abbreviated AOT) in different nonpolar media. AOT has a well-known v-shaped molecular geometry, giving rise to stable reverse micelles without co-surfactants. The reverse micellar solution can dissolve large amount of water with the formation of discrete droplet reverse micelles or bicontinuos reverse micelles. ${ }^{3,4}$ The size of the droplets depends only on water concentration defined as the water-tosurfactant molar ratio $\mathrm{W}_{0}\left(\mathrm{~W}_{0}=\left[\mathrm{H}_{2} \mathrm{O}\right] /[\mathrm{AOT}]\right)$. The micellar pseudophase becomes a nanoreactor the size of which can be readily varied by choosing the experimental conditions, such as the concentration of surfactant and water, the strength of surfactant-water interaction, the size of the water pool, the microscopic viscosity and the polarity of the aqueous interior control. ${ }^{4,5}$ The effect of AOT reverse micelles on a wide variety of chemical reactions has been recently reviewed. ${ }^{3}$ Presently the kinetic models that allow interpreting intrinsic reactivity constants in these media are still scarce. ${ }^{6}$

For a number of years we have been interested in bimolecular aromatic nucleophilic substitutions (SNAr) reactions between several nitro substituted aromatic substrates and aliphatic amines in n-hexane ${ }^{7.8}$ and other non-polar ${ }^{9}$ and polar aprotic solvents. ${ }^{10.11}$ For the $\mathrm{S}_{\mathrm{N}} \mathrm{Ar}$ reactions involving primary or secondary amines the addition-elimination mechanism is commonly found even though a dimer mechanism was also found. ${ }^{12.13}$

The mechanism of the amino dechlorination reaction between 1-chloro-2,4-dinitrobenzene (CDNB) and n-butyl amine (NBA) and piperidine (PIP) in pure n-hexane was extensively study and reported to be base catalyzed. ${ }^{11}$ The magnitude of the catalysis depends on the structure of the amine being noteworthy in the case of NBA.

In micellar systems, the overall rate constant dependence with the surfactant concentration can be quantitatively treated taking into account the species distribution between the micellar pseudophase and the bulk organic solvent, assuming that the incorporation of each reactant is not affected by the presence of the other, ${ }^{14}$ and that the $\mathrm{S}_{\mathrm{N}} \mathrm{Ar}$ reaction is rate limiting. Under this hypothesis the reaction kinetics is pseudo first order in the aromatic substrate. Thus, the partition coefficients for each reactant can be kinetically obtained and the intrinsic $S_{N} A r$ rate constant in the organic phase can be evaluated.

The $\mathrm{S}_{\mathrm{N}} \mathrm{Ar}$ reaction of CDNB and anion of glutathione in various water-surfactant - isooctane reverse micellar systems has been reported. ${ }^{15,16}$ The authors conclude that the cationic surfactant (cetyltrimethylammonium bromide) in isooctane mimics the active site of glutathione transferase and is capable of stabilizing the negatively charged Meisenheimer intermediate catalyzing the reaction. Reverse micellar catalysis for the decomposition of Meisenheimer complexes, intermediates of $\mathrm{S}_{\mathrm{N}} \mathrm{Ar}$, were also reported in several micellar systems but mostly using cationic surfactants. $^{17}$ 
For the $\mathrm{S}_{\mathrm{N}} \mathrm{Ar}$ reaction of 1-fluoro-2,4-dinitrobenzene (FNDB), with hydroxide ion and aliphatic amines in reverse micelle media it was found that, ${ }^{18-19,20}$ compared to a homogeneous phase, cationic micelles catalysed the reaction involving both nucleophiles, whereas inhibition was observed with anionic nucleophiles and negatively charged micelles. Electrostatic attractive interactions between the nucleophiles or the rate limiting transition state with the positively charged interface were claimed to be the responsible for the observed increase in the reactivity. The opposite is true when dealing with anionic surfactants. ${ }^{18}$ For the reaction of FDNB with neutral nucleophiles in AOT/n-hexane/water micelles faster reactions were found in comparison with n-hexane. The rate of reactions depends on $\mathrm{W}_{\mathrm{o}}$. The reaction, which is wholly base catalysed in n-hexane, changes the mechanism in the micelle interface, with the formation of the sigma intermediate the rate determining step. ${ }^{19}$ In AOT/benzene/water micellar systems with a neutral nucleophile, the reactivity is slightly higher than in pure benzene at low nucleophile concentration. At high nucleophile concentration, inhibition was observed with respect to pure benzene. Using benzyl-n-hexadecyl dimethylammonium chloride as surfactant the reaction was accelerated compare with the rate in benzene

In this work we extend the kinetic study to the reaction of a related substrate CDNB, less prone to base catalysis than FNDB, with n-butyl amine (NBA) and piperidine (PIP). The effect of the nucleophile, surfactant concentration and the amount of water in the system are shown, and a plausible mechanism for the SNAr reaction is proposed. The difference in the micellar effects observed changing the leaving group from chloride to fluoride is also discussed.

\section{Results and Discussion}

The reactions of CDNB with NBA and PIP, as observed in pure n-hexane, give the ipso-chloro substitution product in AOT/n-hexane/water reverse micelles (eqn. 1). The UV-visible spectroscopic analysis of the reaction mixture at infinite time shows the corresponding alkyl anilines, N-butyl-2,4-dinitroaniline and N-piperidyl-2,4-dinitroaniline in quantitative yields. This fact was confirmed by HPLC and GC-MS chromatographic analysis.

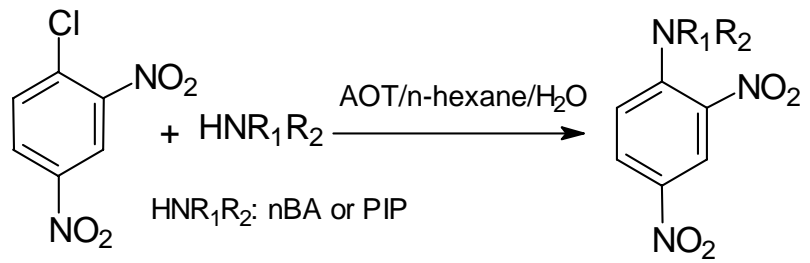

In every case a large excess of nucleophile was used and the reactions followed a pseudofirst-order kinetics. It should be pointed out that no amine-catalyzed hydrolysis was detected at the micelle interface. Moreover, the UV-visible spectroscopic analyses of the reactions at different times show a clear isosbestic point evidencing the cleanness of the reaction. It seems 
that the excess of amine used and the buffer like character of these reverse micelles ${ }^{21}$ precludes the interference that hydroxide ions promoted by amine hydrolysis may cause.

Previous studies have shown that in n-hexane, the reaction is base catalyzed while in aromatic solvents only mild acceleration was observed. The competitive formation of an electron-donor-acceptor complex (EDA) between the reactants or the substrate with donor solvents prior to the sigma intermediate formation was suggested to explain the difference in reactivities. $^{11}$ A similar proposal has been made for other nonpolar aprotic solvents. ${ }^{22.23}$ However, since the role of the EDA complex is kinetically undistinguishable under the reaction conditions no point will be made on this aspect. In pure n-hexane base catalysis was clearly observed in the case of NBA while with PIP only a mild acceleration was observed. Studies with non-nucleophilic bases showed it was genuine catalysis instead of a medium effect. ${ }^{11}$

\section{Effects of AOT concentration}

The kinetics of these reactions were studied at different AOT concentrations, keeping the other experimental conditions constant. The studies were performed at $\mathrm{W}_{\mathrm{o}}=0$ and 10 and shown in Figures 1 and 2 .

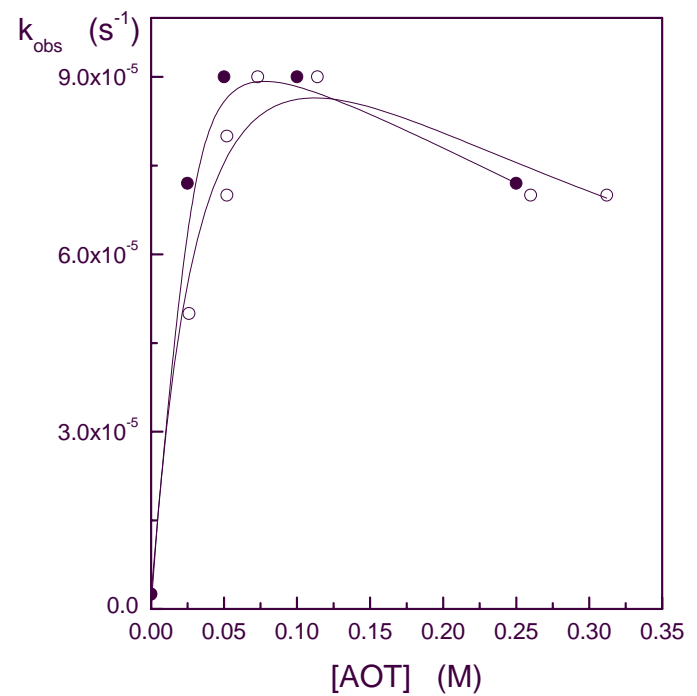

Figure 1. Dependence of the pseudo-first order rate constant $\left(\mathrm{K}_{\mathrm{obs}}\right)$ on the AOT concentration, for the reaction between CDNB and NBA in AOT/n-hexane/water reverse micelles. (o) $\mathrm{W}_{0}=0 ;(\bullet) \mathrm{W}_{0}=10$. $[\mathrm{CDNB}]=5.4 \times 10^{-4} \mathrm{M},[\mathrm{NBA}]=2.0 \times 10^{-2}$ $\mathrm{M}$. The solid lines show fitting by eq 4 .

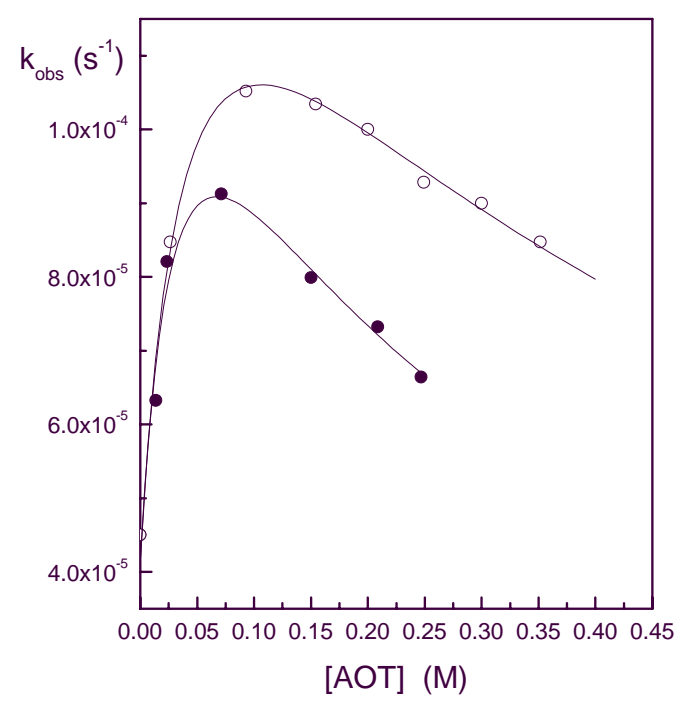

Figure 2. Dependence of the pseudo-first order rate constant $\left(\mathrm{K}_{\mathrm{obs}}\right)$ on the AOT concentration, for the reaction between CDNB and PIP in AOT/ n-hexane/water reverse micelles. (o) $\mathrm{W}_{0}=0 ;(\bullet) \mathrm{W}_{0}=10$. $[\mathrm{CDNB}]=4.5 \times 10^{-4} \mathrm{M},[\mathrm{PIP}]=2.0 \times 10^{-3} \mathrm{M}$. The solid lines show fitting by eq 4 . 
As can be observed $\mathrm{k}_{\text {obs }}$ goes through a maximum (at [AOT] 0.1M) with AOT concentration. Taking into account that when $\mathrm{W}_{\mathrm{o}} \leq 10$ two pseudophases are mainly present, the reaction should be limited to the $n$-hexane phase and the micellar interface. ${ }^{4}$ As can be observed in Figures 1 and 2 , the respective $\mathrm{k}_{\mathrm{obs}}$ in the reverse micelles are at the most twice or three times higher than in pure $n$-hexane for the same amine concentration. The plots in Figures 1 and 2 are characteristic for bimolecular reactions, ${ }^{24}$ that is, the rate of the reaction goes through a maximum in micelles. The initial increase on $\mathrm{k}_{\mathrm{obs}}$ values with the surfactant concentration could be due to the gradual incorporation of the reactants into the micellar aggregates. At higher AOT concentrations the increase in the number of micelles decreases the probability of reactants encounter and rate of reaction decreases. It should also be noted that the maximum value of $\mathrm{k}_{\mathrm{obs}}$ for NBA is less sensitive to $\mathrm{W}_{\mathrm{o}}$ than in the reaction for PIP.

Taking into consideration the higher polarity within the reversed micelles of AOT / n-hexane compare to pure $n$-hexane, ${ }^{25}$ it can be assumed that these reactions do not undergo base catalysis in the micellar interface. Therefore, the experimental data could be explained by means of a mechanism that takes into account the distribution of the reactants between the micellar pseudophase and the bulk n-hexane, and the existence of two-reaction path, one in each phase, as it is summarized by the Scheme 1:

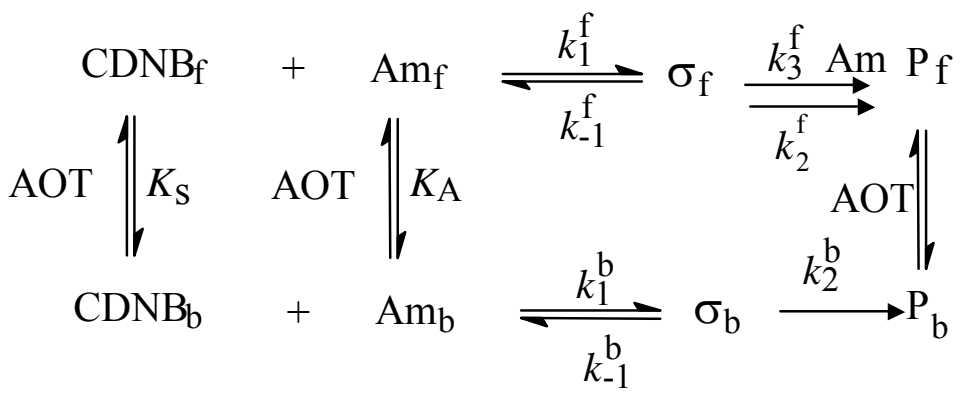

\section{Scheme 1}

where Am represents the nucleophile, while subscripts $\mathrm{f}$ and $\mathrm{b}$ indicates the free and bound reactants in the organic phase and in the micellar pseudophase respectively, and AOT represents the surfactant molecules. The $\mathrm{k}_{\mathrm{i}}{ }^{\mathrm{f}}$ and $\mathrm{k}_{\mathrm{i}}{ }^{\mathrm{b}}$ are the respective rate coefficients for each reaction step in each pseudophase. $\mathrm{K}_{\mathrm{s}}$ and $\mathrm{K}_{\mathrm{A}}$ are the distribution constants between the organic phase and micellar pseudophase for the substrates and the amine, respectively. In pure n-hexane, the reaction is base catalyzed while in polar solvents the reaction is not catalyzed and the formation of the intermediate $\sigma$ is rate limiting. ${ }^{26}$ 
The expressions for the distribution constants are given in eq 2 and 3.

$$
\begin{gathered}
\mathrm{Ks}=\frac{\left[\mathrm{CDNB}_{\mathrm{b}}\right]}{\left[\mathrm{CDNB}_{\mathrm{f}}\right][\mathrm{AOT}]} \\
\mathrm{K}_{\mathrm{A}}=\frac{\left[\mathrm{Am}_{\mathrm{b}}\right]}{\left[\mathrm{Am}_{\mathrm{f}}\right][\mathrm{AOT}]}
\end{gathered}
$$

A simple mass balance using $\mathrm{K}_{\mathrm{S}}$ and $\mathrm{K}_{\mathrm{A}}$ and the analytical concentration of $\mathrm{CDNB}$ and amine respectively allow us to calculate their respective interface concentrations.

According to the proposed mechanism (Scheme), assuming a steady state for the $\sigma$ intermediates, and taking into account the reaction molar volume at the interface, which can be estimated from the molar volume of AOT in the reverse micelles, ${ }^{27}$ the rate of the reaction can be expressed by eq 4. where the concentrations in square brackets are the analytical concentrations and refer the total volume of reverse micelle.

$$
\mathrm{k}_{\mathrm{obs}}=\frac{\left(\mathrm{k}^{\prime}\left(1+\mathrm{K}_{\mathrm{A}}[\mathrm{AOT}]\right)+\mathrm{k}^{\prime \prime}\left[\grave{A}_{\mathrm{t}}\right]\right)\left(\mathrm{Am}_{\mathrm{t}}\right]}{\left(1+\mathrm{K}_{\mathrm{S}}[\mathrm{AOT}]\right)\left(1+\mathrm{K}_{\mathrm{A}}[\mathrm{AOT}]\right)^{2}}+\frac{\mathrm{k}_{1}^{\mathrm{b}} \mathrm{K}_{\mathrm{s}} \mathrm{K}_{\mathrm{A}}[\mathrm{AOT}]\left[\mathrm{Am}_{\mathrm{t}}\right] / \overline{\mathrm{v}}}{\left(1+\mathrm{K}_{\mathrm{S}}[\mathrm{AOT}]\right)\left(1+\mathrm{K}_{\mathrm{A}}[\mathrm{AOT}]\right)}
$$

where $\mathrm{k}^{\prime}=\frac{\mathrm{k}_{1}^{\mathrm{f}} \mathrm{k}_{2}^{\mathrm{f}}}{\mathrm{k}_{-1}^{\mathrm{f}}}$ and $\mathrm{k}^{\prime \prime}=\frac{\mathrm{k}_{1}^{\mathrm{f}} \mathrm{k}_{3}^{\mathrm{f}}}{\mathrm{k}_{-1}^{\mathrm{f}}}$.

The distribution constant of CDNB between n-hexane and the micellar interface at different $\mathrm{W}_{0}$ values was evaluated independently by using the fluorescence quenching method proposed by Encinas and Lissi. ${ }^{28}$ Tris(2,2'-bipyridine)ruthenium (II), which is quenched by nitro aromatic compounds, was used as the fluorescence probe. ${ }^{29}$ The distribution constant of CDNB in microemulsion solutions was defined by eq. 2 . Thus, the substrate is only partitioned between the micellar interface and the organic solvent, and completely excluded from the water pool.

A value of $\mathrm{K}_{\mathrm{S}}$ of $20 \pm 2 \mathrm{dm}^{3} \mathrm{~mol}^{-1}$ independent on the $\mathrm{W}_{0}$ was obtained as previously observed for 1-fluoro-2,4-dinitrobenzene ${ }^{18}$

\section{Kinetic parameter evaluation}

The measured $\mathrm{K}_{\mathrm{S}}$ value and a molar AOT volume of $0.38 \mathrm{~L} / \mathrm{mol}$ were introduced in the fitting equation. The values of $\mathrm{k}^{\prime}$ and $\mathrm{k}$ " used, were previously reported for the reaction in neat organic solvent ( 0 and $4 \times 10^{-5}$ for NBA, and $2.05 \times 10^{-2}$ and 0.118 for PIP respectively). ${ }^{11}$ The intrinsic rate constant for the reaction at the interface $\mathrm{k}_{1}{ }^{\mathrm{b}}$ and $\mathrm{K}_{\mathrm{A}}$, were obtained by non-linear least square fitting of the $\mathrm{k}_{\mathrm{obs}}$ versus [AOT] plots by means of eq. 4 . The generated curves, shown in Figures 1 and 2, agree quite closely with the experimental points. The kinetic parameters obtained are collected in Table 1 . The values of $\mathrm{K}_{\mathrm{A}}$ calculated with these fittings are very similar 
to the obtained for the reaction of FNDB with the same amines in the same micellar media, ${ }^{19}$ which is a good indication that the proposed mechanism is operative.

As can be observed in Figures 1 and 2, the reaction rate in the micellar medium is twice the value obtained in pure n-hexane. A greater effect, two orders of magnitude higher, was reported when fluorine is the leaving group ${ }^{19}$ As in pure n-hexane, the reaction involving chlorine, a better leaving group than fluorine, is less prone to nucleophilic or micellar catalysis and also less sensitive to micelle concentrations. Taking into account that the lowest AOT concentration used is far above the cmc, ${ }^{30}$ these results seem to indicate that in reverse micelles the nucleophilic aromatic substitution reaction takes place mainly at the AOT interface.

As can be observed in Table 1, the distribution constants for the amines are smaller than that of CDNB. Taking into account the higher lipophilicity of the later, the hydrophobicity seems not to be the responsible for the observed order ( $\log \mathrm{P}$ values are $0.59,0.52$ and 2.47 for NBA, PIP and CDNB respectively). ${ }^{31}$ The higher dipole moment of CDNB compared to that of NBA and PIP, might play an important role in the partition $\left(4.86,0.36\right.$ and $0.38 \mathrm{D}$ respectively). ${ }^{32}$ It can also be noted that NBA distribution constant is slightly higher than the one of PIP, probably due to the presence of second hydrogen for hydrogen bond interaction with the polar interface. In any case, the ratio between both distribution constants is almost independent of the amount of water in the micelle, $\mathrm{W}_{0}$.

Table 1. Kinetics parameters for the reaction of CDNB with NBA and PIP in AOT-n-hexane at different $\mathrm{W}_{0}$

\begin{tabular}{lcccc}
\hline Amine & $\mathrm{W}_{0}$ & $10^{3} \mathrm{k}_{1}^{m}\left(\mathrm{~s}^{-1} \mathrm{M}^{-1}\right)$ & $\mathrm{K}_{\mathrm{A}}\left(\mathrm{M}^{-1}\right)$ & $n$ \\
\hline NBA & 0 & $1.0 \pm 0.1^{a}$ & $3.3 \pm 0.4$ & 9 \\
& 10 & $0.6 \pm 0.1^{b}$ & & 10 \\
& & $0.6 \pm 0.2^{b}$ & $8.7 \pm 0.5$ & 11 \\
& 0 & $12.8 \pm 0.6^{a}$ & $2.7 \pm 0.2$ & 8 \\
PIP & $14 \pm 2^{b}$ & & 7 \\
& 10 & $6.1 \pm 0.5^{a}$ & $6.0 \pm 0.7$ & 7 \\
& & $5.9 \pm 0.2^{b}$ & & 6 \\
\hline
\end{tabular}

${ }^{a}$ From fitting a plot $\mathrm{k}_{\text {obs }}$ vs [AOT] by eq. $4 .{ }^{\mathrm{b}}$ From fitting a plot $\mathrm{k}_{\text {obs }}$ vs [Am ] by eq. 4; parameters values calculated using the 0.995 confidence level in the non-linear regression. $\mathrm{n}$ : number of points. 


\section{Effect of amine concentration}

In order to check the proposed mechanism, the effect of the change in amine concentration upon reactivity was investigated. Thus, the reaction kinetics at constant AOT concentration $(0.2 \mathrm{M}$ AOT) at $\mathrm{W}_{0}=0$ and 10 were measured. The chosen AOT concentration is slightly higher than the one needed to obtain the maximum rate in Figures 1 and 2 . The results are shown in Figure 3 and 4 . The observed linear relationships indicate that the reaction at the polar micellar interface is not base catalyzed.

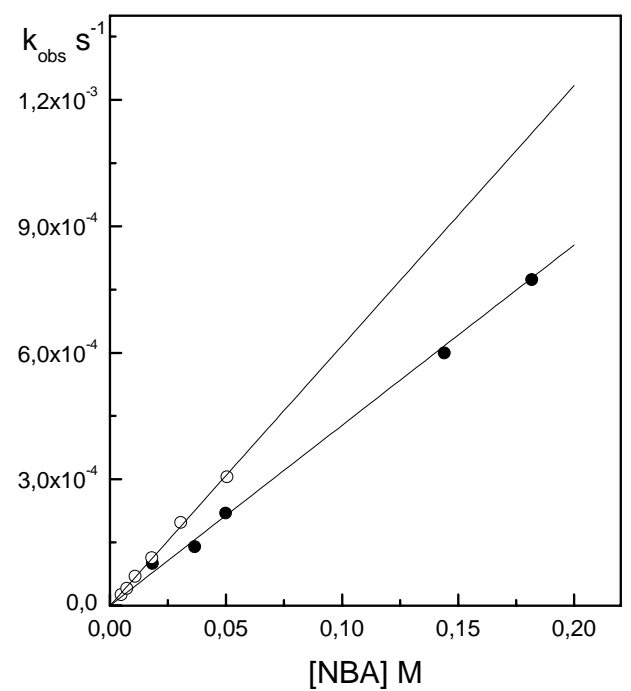

Figure 3. Variation of the pseudo-first-order rate constant $\left(\mathrm{K}_{\mathrm{obs}}\right)$ with NBA concentration, for the reaction between $\mathrm{CDNB}$ and NBA in AOT/ n-hexane/water reverse micelles. (o) $\mathrm{W}_{0}=0 ;(\bullet) \mathrm{W}_{0}=10 .[\mathrm{CDNB}]=2.5 \times 10^{-4}$ $\mathrm{M},[\mathrm{AOT}]=0.2 \mathrm{M}$. The solid lines show fitting by eq 4 .

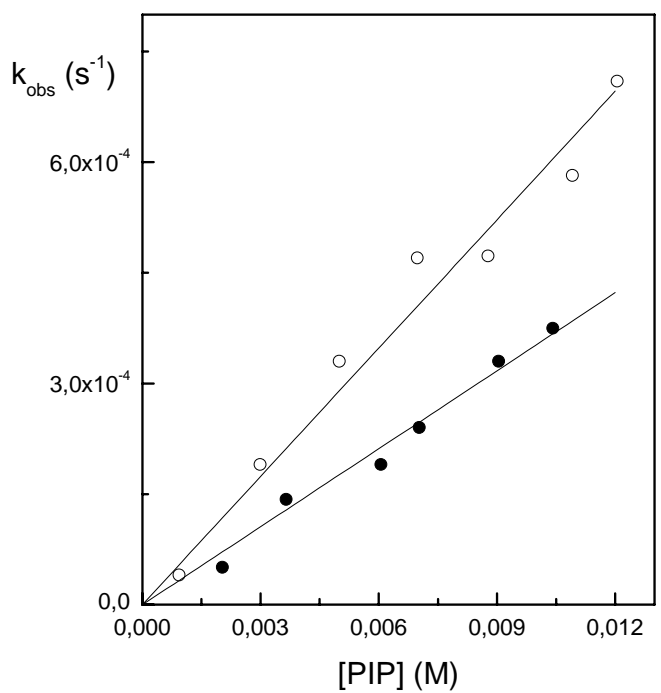

Figure 4. Variation of the pseudo-first-order rate constant $\left(\mathrm{K}_{\mathrm{obs}}\right)$ with PIP concentration, for the reaction between CDNB and PIP in AOT/ n-hexane/water reverse micelles. (o) $\mathrm{W}_{0}=0 ;(\bullet) \mathrm{W}_{0}=10 .[\mathrm{CDNB}]=7.7 \times 10^{-4}$ $\mathrm{M},[\mathrm{AOT}]=0.2 \mathrm{M}$. The solid lines show fitting by eq 4.

By introduction of the proper $\mathrm{K}_{\mathrm{A}}$ values, the values of $\mathrm{k}_{1}{ }^{\mathrm{b}}$ were estimated by fitting the new set of experimental data (Figure 3 and 4) by eqn. 4. The results are included in Table 1. As can be seen, good estimates within experimental error for $\mathrm{k}_{1}{ }^{\mathrm{b}}$ were obtained by two independent data sets.

Thus, the kinetic results are consistent with the assumption that, in AOT/n-hexane/water reverse micelles, these reactions are not base catalyzed and the formation of $\sigma$ intermediate is rate determining. In fact, in this micellar system the surfactant polar heads have shown strong hydrogen bond acceptor ability ${ }^{33}$ and they may be assisting the intermediate decomposition to products. On the other hand, the reaction in the organic solvent is base catalyzed in both cases. 
As expected, PIP, the best nucleophile, reacts one order of magnitude faster than NBA (Table 1). This result agrees with the findings for $S_{N} A r$ reactions in which the formation of the intermediate $\sigma$, is rate determining. ${ }^{34,35,19}$ The higher reactivity of secondary over primary amine has been adduced to be due to a favorable ion-induced dipole interaction in the transition state between partially the positively charged amine nitrogen and the polarizable alkyl moieties attached to it. ${ }^{35}$

\section{Effect of amount of water dispersed}

As can be observed in Table 1, the amine distribution constants increase with the amount of water in the micellar pseudo-phase, probably due to the high water solubility of the amines. As anticipated in the study of effect the AOT concentration on the reactivity, the effect of micellar water content on the reactivity of NBA is not noticeable. However, the reaction involving PIP was sensitive to water addition (Figure 5) particularly for values of $\mathrm{W}_{0}<5$.

Even though it is known the existence of the so-called "built solvation" between the amino hydrogen atoms and the o-nitro group in the zwitterionic form of the $\sigma$ intermediate, ${ }^{36}$ in the case of NBA, the presence of an extra hydrogen atom gives rise to a strong hydrogen-bond donor transition state. Since the reaction is occurring in the waterside of the interface in the case of NBA both the nucleophile and the transition state are strongly solvated. In the case of PIP, the nucleophile solvation competes with the strong intramolecular built in solvation in the transition state and the effect will be more noticeable at low water content where the AOT polar heads are being hydrated.

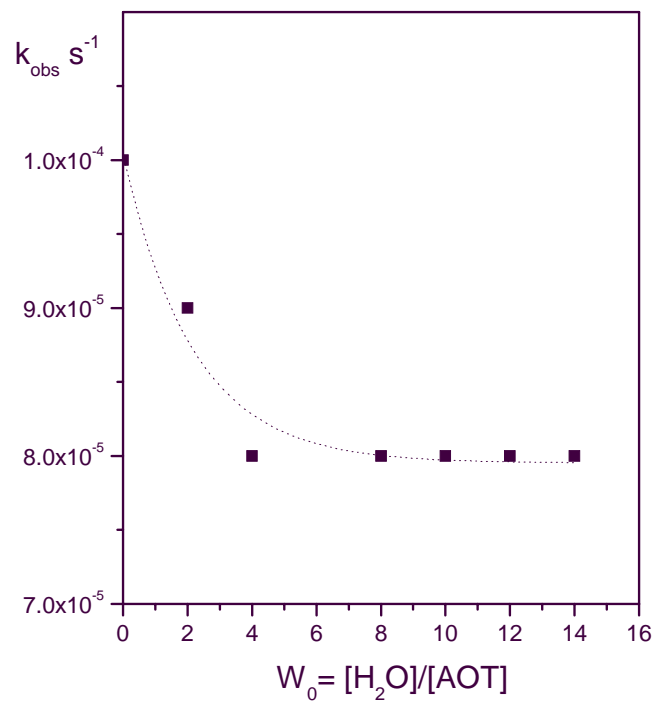

Figure 5. Variation of the pseudo-first-order rate constant $\left(\mathrm{K}_{\mathrm{obs}}\right)$ with $\mathrm{W}_{0}$ in $\mathrm{AOT} / \mathrm{n}$ hexane/water reverse micelles, for the reaction CDNB with PIP. [CDNB] $=1.6 \times 10^{-4} \mathrm{M},[\mathrm{PIP}]$ $=2.0 \times 10^{-3} \mathrm{M},[\mathrm{AOT}]=0.2 \mathrm{M}$. 


\section{Conclusions}

The overall rate of the reaction of CDNB with NBA and PIP are slightly accelerated in the reverse micelle medium with respect to the pure solvent. In both cases the reaction goes through a maximum at AOT concentration around $0.1 \mathrm{M}$. These results suggest a change in the ratelimiting step of the reaction when shifting from pure solvent to micellar medium. Thus, in nhexane the reaction is base catalyzed, while in reverse micelle medium, the polar surfactant heads at the interface may assist the $\sigma$ intermediate decomposition. The kinetic behavior can be quantitatively explained taking into account the distribution of the reactants between the bulk organic solvent and the micellar pseudo-phase. The results were used to evaluate the amine distribution constant and the intrinsic second-order rate coefficient of $\mathrm{S}_{\mathrm{N}} \mathrm{Ar}$ reaction at the interface. At the same value of $\mathrm{W}_{0}$, PIP reacts faster than NBA as normally observed in these types of reactions. However, the observed rates for NBA are almost independent of $\mathrm{W}_{0}$ while for PIP a slight decrease at $\mathrm{W}_{0}=10$ was observed. The different behavior was explained in term of the difference in hydrogen bond donor ability of the $\sigma$ intermediates.

The present results can be used as a good model to predict micellar catalysis for reaction between nonionic substrates, which give zwitterionic intermediates.

\section{Experimental Section}

General Procedures. UV-visible spectra were recorded on a Hewlett-Packard HP 8452 spectrophotometer. Materials. 1-Chloro-2,4-dinitrobenzene (CDNB) from Aldrich, n-butylamine (NBA) and piperidine (PIP) from Riedel-deHaën were used without further purification. Sodium bis(2-ethylhexyl) sulfosuccinate (AOT) from Sigma was dried under vacuum over $\mathrm{P}_{2} \mathrm{O}_{5}$. nHexane (Sintorgan, HPLC quality) were used as received. Ultra pure water was obtained from a Labconco equipment model 90901-01.

\section{Procedures}

Stock solutions of AOT reverse micelle were prepared by weighing and dilution in n-hexane. Stock solution of $1 \mathrm{M}$ AOT was agitated in a sonicating bath until the reverse micelle was optically clear. The appropriate amount of stock solution to obtain a given concentration of AOT in the micellar media was transferred into the cell. The addition of water to the corresponding solution was performed using a calibrated microsyringe. The amount of water present in the system was expressed as the molar ratio between water and the surfactant present in the reverse micelle $\left(\mathrm{W}_{0}=\left[\mathrm{H}_{2} \mathrm{O}\right] /[\mathrm{AOT}]\right)$. The $\mathrm{W}_{0}=0$ correspond to a system with no addition of water and its presence corresponds to the intrinsic humidity of the system $\left(\mathrm{W}_{0} \cong 0.3\right)$. 


\section{Kinetics}

Reactions were performed by following spectrophotometrically the increase in the maximum absorption band of the product, $\mathrm{N}$-alkyl-2,4-dinitroaniline, at $25.0 \pm 0.5^{\circ} \mathrm{C}$. To start a kinetic run, a stock solution of CDNB was added $(10 \mu \mathrm{l})$ into a thermostated cell containing the reverse micelle solution. The CDNB concentration was $1.5-5.0 \times 10^{-4} \mathrm{M}$. The kinetic runs were performed following the increasing in the absorbance of the product of the reaction at 360 and $392 \mathrm{~nm}$ for PIP and NBA respectively. In all the cases, pseudo-first-order plots were obtained in excess of nucleophile. The pseudo-first-order rate constants $\left(\mathrm{k}_{\mathrm{obs}}\right)$ were obtained by a non-linear leastsquares fit of the experimental data, absorbance vs. time, by a first order rate equation $(\chi 2<$ $0.05)$. The value of the absorbance at infinite reaction time was consistent with the value obtained from authentic samples of the reaction product, within 3\%.

\section{Acknowledgments}

We gratefully acknowledge the financial support for this work by the Consejo Nacional de Investigaciones Científicas (CONICET), Secretaría de Ciencia y Técnica de la Universidad Nacional de Río Cuarto and Agencia Nacional de Promoción Científica y Tecnológica. SMC and JJS hold a research position at CONICET.

\section{References}

1. Fendler, J.; Fendler, J. H. Catalysis in Micellar and Macromolecular Systems; Acad. Press: New York, 1975.

2. Pileni, M. P. Structure and Reactivity of Reverse Micelles; Elsevier: New York, 1989.

3. De, T. K.; Maitra, A. Adv. Colloid Interface Sci. 1995, 59, 95.

4. Pileni, M. P. J. Phys. Chem. 1993, 97, 6961.

5. García-Río, L. Leis, J. R. Iglesias, E. J. Phys. Chem. 1995, 99, 12318.

6. Blagoeva, I. B.; Gray, P.; Ruasse, M-F. J. Phys. Chem. 1996, 100, 12638.

7. Chiacchiera, S. M.; Singh, J. O.; Anunziata, J. D.; Silber, J. J. J. Chem. Soc., Perkin 2 1987, 987.

8. Cattana, R. S.; Singh, J. O.; Anunziata, J. D.; Silber, J. J. J. Chem. Soc. Perkin, 2 1987, 79.

9. Chiacchiera, S. M.; Singh, J. O.; Anunziata, J. D.; Silber, J. J. J. Chem. Soc. Perkin 2 1988, 1585.

10. Chiacchiera, S. M.; Cattana, R. I. ; Singh, J. O.; Anunziata, J. D.; Silber, J. J. J. Phys. Org. Chem. 1989, 2, 631.

11. Durantini, E. N.; Zingaretti, L.; Anunziata, J. D.; Silber, J. J. J. Phys. Org. Chem. 1992, 2, 557.

12. Terrier, F. Nucleophilic Aromatic Displacement, VCH Publishers, Inc.: New York, 1991. 
13. Nudelman, N. S. The Chemistry of amino, nitroso, nitro and related groups, Patai, S., Ed.; Wiley: New York, 1996, Ch. 26.

14. Iglesias, E.; Leis, J. R.; Peña, M. E.; Langmuir 1994, 10, 662.

15. Tang, S.-S.; Chang, G.-G.; J. Org. Chem. 1995, 60, 6183.

16. Liou, J.-Y.; Huang, T.-M; Chang, G.-G.; J. Chem. Soc., Perkin Trans 2 1999, 2171.

17. Fendler, J. H. Acc. Chem. Res. 1976, 9, 153.

18. Durantini, E. N.; Borsarelli; C. D.; J. Chem. Soc., Perkin 2 1996, 719.

19. Correa, M. N.; Durantini, E. N.; Silber, J. J. J. Org. Chem. 1999, 64, 5757.

20. Correa, M. N.; Durantini, E. N.; J. J. Silber, J. Org. Chem. 2000, 65, 6427.

21. Hasegawa, M.; Langmuir 2001, 17, 1426.

22. Forlani, L.; Bosi, M. J. Phys. Org. Chem. 1992, 5, 429.

23. Forlani, L. J. Chem. Soc., Perkin 2 1993, 1525.

24. O'Connor, C. J.; Lomax, T. D.; Ramage, R. E. In Solution behavior of surfactants: theoretical and applied aspects; Mittal, K. L.; Fendler, E. J. Eds.; Plenun Press: New York, 1982, Vol 2, p 803.

25. Correa, N. M.; Biasutti, M. A.; Silber, J. J. J. Colloid and Interface Sci. 1995, 172, 71.

26. Bernasconi, C. F. M. T. P. Int. Rev. Sci. Org. Chem. Ser. One 1973, 3, 33.

27. García-Río, L. Ramón Leis, J. M. Peña, E. Iglesias, E. J. Phys. Chem. 1993, 97, 3437.

28. (a) Encinas M. V. Lissi, E. A. Chem. Phys. Lett. 1982, 91, 55. (b) Encinas M. V. and Lissi, E.

A. Chem. Phys. Lett. 1986 132, 545. (c) Lissi, E. A.; Encinas, M. V.; Alvarez, J.; Borsarelli, C. D., J. Colloid Interface Sci. 1993, 160, 472.

29. Bock, C. R.; Connor, J. A.; Gutierrez, A. R.; Meyer, T. J. ; Whitten, D. G.; Sullivan, V. P.; Nagle, J. K. J. Am. Chem. Soc. 1979, 101, 4815.

30. Biasutti, M. A.; Correa, N. M.; Silber, J. J. Molecular Interactions in Reverse Micelles. Current Topics in colloid \& interface Science. Research Trends, Goñi, F. M.; Hoffmann, H., Eds.; India 3, 1999, p 35.

31. Estimated by $\log \mathrm{P}$ calculated by Chem Plus ${ }^{\mathrm{TM}}$ Version 1.6 on geometry optimized structures by means of AM1, performed with Hyperchem ${ }^{\mathrm{TM}}$ Pro release 5.1, from Hypercube INC. Estimated Log $\mathrm{P}$

32. Gas Phase dipole moments estimated for geometry optimized structures by means of AM1, performed with Hyperchem ${ }^{\mathrm{TM}}$ Pro release 5.1, from Hypercube INC.

33. Correa, N. M.; Silber, J. J. J. Mol. Liq. 1997, 72, 163.

34. Miller, J. Aromatic Nucleophilic Substitution; Elsevier: Amsterdam, 1968.

35. Bunnet, J. F.; Sekiguchi, S. L.; Smith, A. J. Am. Chem. Soc. 1981, 103, 4865.

36. Bunnet, J. F.; Morath, R. J. J. Am. Chem. Soc. 1955, 77, 5051. 